\title{
Persistent Brain Network Homology from the Perspective of Dendrogram
}

\author{
Hyekyoung Lee, Hyejin Kang, Moo K. Chung*, Bung-Nyun Kim, and Dong Soo Lee
}

\author{
University of Wisconsin-Madison \\ Department of Biostatistics and Medical Informatics \\ Technical Report \# 228 \\ (To appear in IEEE Transactions to Medical Imaging) \\ email: mkchung@wisc.edu \\ September 7, 2012
}

\begin{abstract}
The brain network is usually constructed by estimating the connectivity matrix and thresholding it at an arbitrary level. The problem with this standard method is that we do not have any generally accepted criteria for determining a proper threshold. Thus, we propose a novel multiscale framework that models all brain networks generated over every possible threshold. Our approach is based on persistent homology and its various representations such as the Rips filtration, barcodes and dendrograms. This new persistent homological framework enables us to quantify various persistent topological features at different scales in a coherent manner. The barcode is used to quantify and visualize the evolutionary changes of topological features such as the Betti numbers over different scales. By incorporating additional geometric information to the barcode, we obtain a single linkage dendrogram that shows the overall evolution of the network. The difference between the two networks is then measured by the Gromov-Hausdorff distance over the dendrograms.

As an illustration, we modeled and differentiated the FDG-PET based functional brain networks of 24 attentiondeficit hyperactivity disorder children, 26 autism spectrum disorder children and 11 pediatric control subjects.
\end{abstract}

Asterisk indicates corresponding author

H. Lee is with the Department of Nuclear Medicine and Department of Brain and Cognitive Sciences, Seoul National University, Seoul, Korea (e-mail: leehk@postech.ac.kr).

H. Kang is with the Department of Nuclear Medicine, Seoul National University, Seoul, Korea (e-mail: hkang211@snu.ac.kr).

M. K. Chung is with the Department of Biostatistics and Medical Informatics, and the Waisman Laboratory for Brain Imaging and Behavior, University of Wisconsin, Madison, WI 53705, USA (e-mail: mkchung@wisc.edu).

B.-N. Kim is with the Devision of Child and Adolescent Psychiatry, Department of Neuropsychiatry, Seoul National University, Seoul, Korea (e-mail:kbn1@snu.ac.kr).

D.S. Lee is with the Department of Nuclear Medicine and WCU Department of Molecular Medicine and Biopharmaceutical Sciences, Seoul National University, Seoul, Korea (e-mail: dsl@snu.ac.kr).

This work was supported by NRF grant funded by the Korea government (MEST) (No. 2011-0030815), by NAP grant funded by the Korean Research Council of Fundamental Science and Technology, and by Brain Research Center of the 21st Century Frontier Research Program (2012K001119). This research was also supported by the WCU Department of Brain and Cognitive Science and by grant No. R31-2008-000-10103-0 from the WCU program of the MEST and the NRF. 


\section{Index Terms}

Functional Brain Network, Rips Complex, Rips filtration, Barcode, Persistent Homology, Single Linkage Dendrogram, Gromov-Hausdorff Distance

\section{INTRODUCTION}

Many functional brain connectivity studies have often focused on verifying the topological characteristics of the network such as the small-worldness, scale-freeness or modularity using well-known graph measures [1], [2], [3], [4], [5], [6], [7], [8], [9], [10].

The connectivity of the human brain, also known as human connectome, is usually represented as a graph consisting of nodes and edges connecting the nodes [11]. The nodes are mainly predefined anatomical regions of interest (ROIs). The edges are determined by various technique such as correlation methods, structural equation modeling or dynamic causal modeling [2], [12], [13], [14], [15], [16], [17].

In the correlation approaches, depending on whether we threshold the correlation at a certain level or not, we obtain either weighted or binary networks [18], [17]. Since the weighted brain network is difficult to interpret and visualize compared to the binary network, the binary brain network has more been often used [19], [20]. However, depending on where to threshold the correlation, the binary network changes. To obtain the proper threshold, the multiple comparison correction over every possible edge can be also applicable [21], [3], [22], [23], [24], [20]. However, depending on what $p$-value to threshold, the resulting graph also changes.

Others tried to control the sparsity of edges in the network. The sparsity of a graph is defined as the ratio of the number of edges to the number of all possible edges [25], [26], [6], [27], [20]. Fixing the sparsity needs an educated guess; therefore, two different networks are compared in the preselected range of sparsity [25], [28], [19]. Since this approach is also problematic, in the end, the two different networks are compared at the maximum sparsity.

Until now, there are not widely accepted criteria for thresholding networks. Instead of trying to come up with a proper threshold for network construction that may not work for different clinical populations or cognitive conditions [20], why not use all networks for every possible threshold? Motivated by this question, we developed a novel multiscale hierarchical network modeling framework that traces the evolution of network changes over different thresholds. Since we are using networks constructed at every threshold, we practically bypass the problem of determining the optimal threshold. However, one main technical huddle of using every possible network at different scales is the inherent computational burden of handling significantly many networks. The persistent homology, a new branch of the algebraic topology, provides a clue for efficiently handling and analyzing multiscale networks by identifying the persistent topological features over changing scales [29], [30], [31].

The concept of persistent homology has been previously applied to medical image analysis [32], [33], [34], [35]. In particular, Singh, et. al. applied the persistent homology to the electrocorticography-based connectivity in primary visual cortex of macaque previously [34]. They tried to find the proper threshold for connectivity matrix using persistent homology. On the other hand, in this paper, we will show that 
it is also possible to do network modeling without determining the threshold within the same persistent homology framework.

The brain network corresponds to the Rips complex, which is the main algebraic representation used in persistent homology, and the multiscale networks corresponds to the Rips filtration, which is the sequence of the nested Rips complexes over different scales [36]. The main topological features are the Betti numbers. Among the Betti numbers, the first three Betti numbers, which count the number of connected components, holes and voids, would be of interest in practice. In this paper, we will mainly focus on the zeroth Betti number $\beta_{0}$, which measures the number of the connected components.

The changes of the Betti numbers over the Rips filtration can be visualized using the barcode [37], [38]. The barcode is a topologically invariant representation of the network change over the filtration. So it does not have geometric information of node positions. If we incorporate the node indexing to the barcode, surprisingly we obtain the single linkage dendrogram (SLD) [39], [40], [41]. Since the distance between two different SLDs can be measured using the Gromov-Hausdorff (GH) distance [40], [41], we can directly measure the distance between any two networks.

The two main contributions of this paper are:

(1) We propose a new multiscale network modeling framework for brain connectivity that avoids using a single fixed threshold. The proposed method basically uses networks generated at every possible threshold. The computational challenge of handling significantly many networks was addressed by introducing the concept of graph filtration in the persistent homology framework.

(2) We show that, if we add the geometrical information of node indexing to the barcode, we obtain SLD. The difference between two different SLDs can be measured using the GH distance. Hence, our method provides the first unified mathematical framework for measuring brain network differences.

The proposed method is applied in differentiating the abnormal resting glucose metabolic networks using 103 ROIs extracted from FDG-PET of 24 attention-deficit hyperactivity disorder (ADHD), 26 autism spectrum disorder (ASD) and 11 pediatric controls (PedCon). Numerical experiments show that our graph filtration framework can differentiate the populations better than most known graph theoretic approaches as well as the previous persistent homology framework.

\section{BRAIN NETWORK CONSTRUCTION}

Consider FDG-PET measurements obtained in $p$ selected ROIs in $n$ subjects. Each ROI serves as a node in the brain network. We have the FDG-PET measurement $x_{i}$ at $i$-th node. The measurement set is denoted as $X=\left\{x_{1}, \cdots, x_{p}\right\}$. The measurement $x_{i}$ is assumed to be normally distributed with mean zero and the variance 1 . This condition can be guaranteed by centering and normalizing the measurements. We define the distance $c_{X}$ between the measurements $x_{i}$ and $x_{j}$ through the Pearson correlation:

$$
c_{X}\left(x_{i}, x_{j}\right)=1-\operatorname{corr}\left(x_{i}, x_{j}\right)
$$

The functional brain network is represented using measurement set $X$ and the distance $c_{X}$, which form the metric space $\left(X, c_{X}\right)$. 


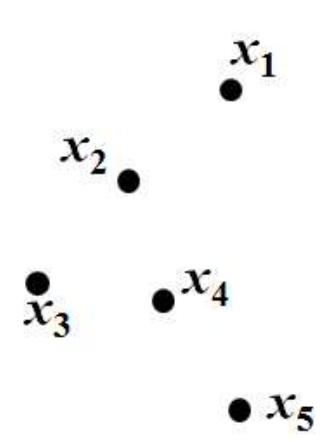

(a)

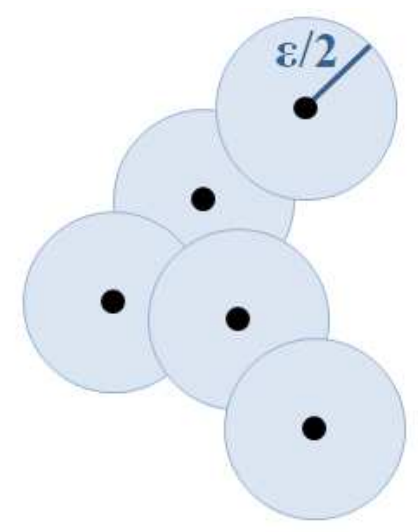

(b)

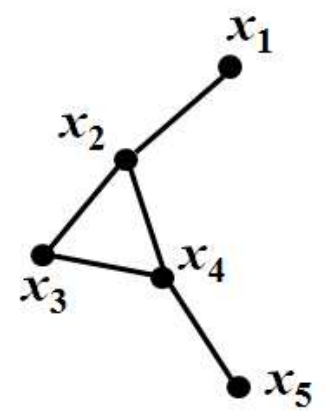

(c)

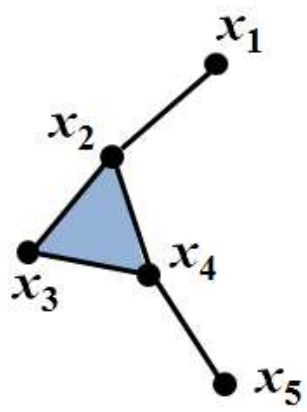

(d)

Fig. 1. Construction of binary network and Rips complex. (a) Point cloud data $X$ (b) The balls of radius $\epsilon / 2$ centered at each point. (c) The binary network $\mathcal{B}(X, \epsilon)$. (d) The Rips complex $\mathcal{R}(X, \epsilon)$.

Let us more formally define the network constructed by thresholding correlations between the nodes.

Definition 1: We connect the nodes $i$ and $j$ with an edge if the distance $c_{X}\left(x_{i}, x_{j}\right) \leq \epsilon$ for some threshold $\epsilon$. The collection of all those edges is denoted as $E$. Then the binary network $\mathcal{B}(X, \epsilon)$ at threshold $\epsilon$ is a graph consisting of the node set $V$ and the edge set $E$.

All previous studies on brain network modeling used the single fixed threshold $\epsilon$ [19], [18], [17], [20] while we are trying to avoid using a fixed threshold using the persistent homology framework.

\section{Persistent Network homology And Clustering}

In this section, we introduce the basic concepts used in persistent homology and relate them to brain network modeling.

\section{A. Network as simplicial complex}

The shape of an object can be approximated by the point cloud data (node set) $X$ consisting of $p$ points. If we connect points of which distance satisfies a given criterion, the connected points start to recover the topology of the object. Therefore, we can represent the underlying topology as a collection of the subsets of $X$ that consist of nodes which are connected [29], [42]. Denote the collection of all possible subsets as $2^{X}$. There are $2^{p}$ possible subsets of $X$ that can be a possible topology. Here we provide the formal definition of the topology [29].

Definition 2: If $\mathcal{U} \subset 2^{X},(X, \mathcal{U})$ is a topological space on the finite set $X$ if

1) $\emptyset, X \subset \mathcal{U}$,

2) $u_{1}, u_{2} \subset \mathcal{U}$ implies $u_{1} \cup u_{2} \subset \mathcal{U}$ and

3) $u_{1} \cap u_{2} \subset \mathcal{U}$.

Note that every metric space is a topological space. Hence, the binary network $\mathcal{B}(X, \epsilon)$ is also a topology. In general, given a point cloud data set $X$ with a rule for connections, the topological space is a simplicial 


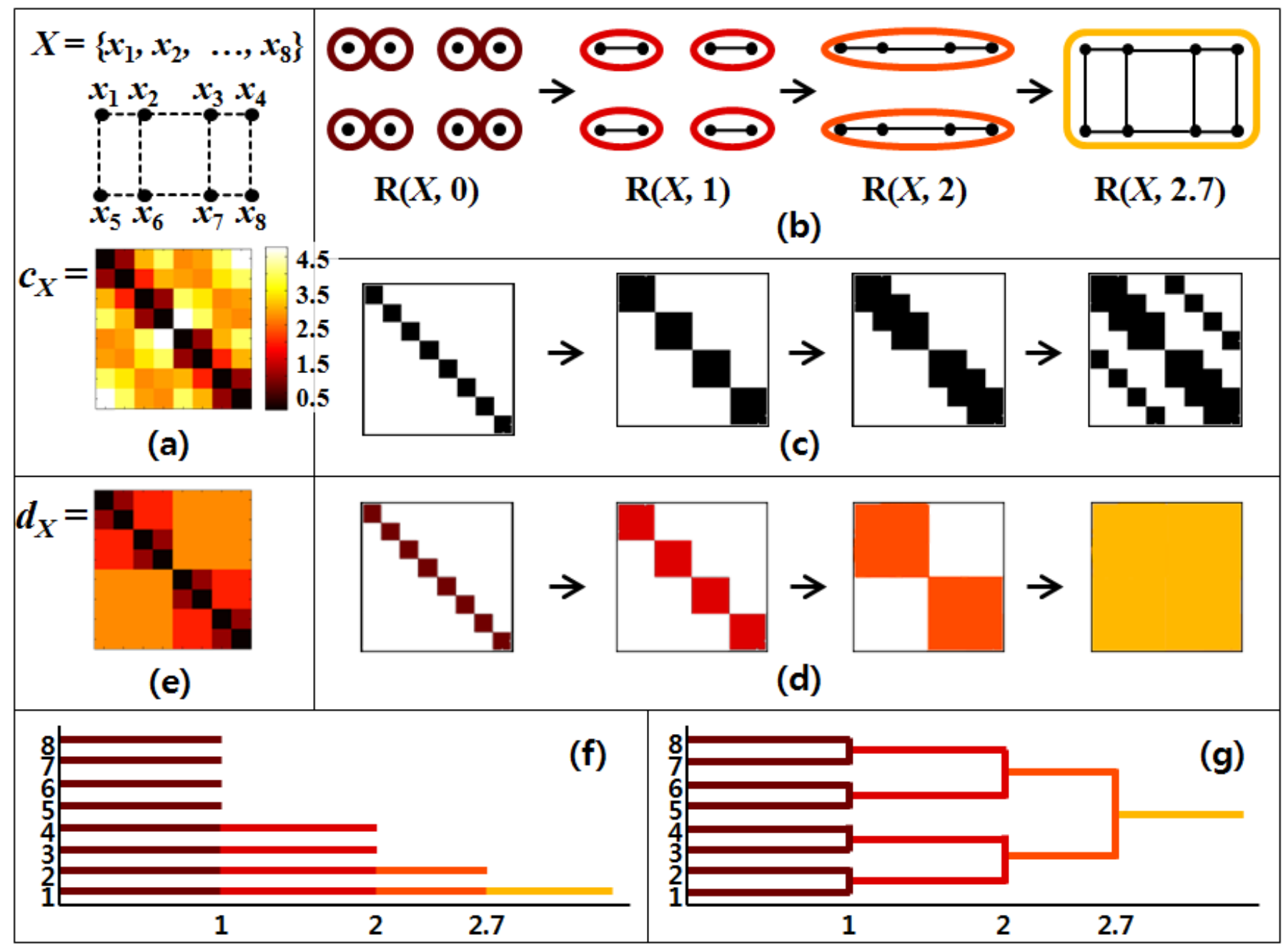

Fig. 2. The schematic of the multiscale network modeling via barcode and dendrogram. (a) Node set $X$ and metric $c_{X}$. (b) The Rips filtration at the filtration values $0,1,2$ and 2.7. (c) The corresponding adjacency matrices of the Rips filtration. (d) The connected component matrices representing the connected components through different colors. (e) The single linkage distance $d_{X}$. (f-g) The topological changes are visualized by the barcode and the single linkage dendrogram (SLD). The vertical axis of the barcode represents the zeroth Betti number $\beta_{0}$, and one of dendrogram represents the indices of nodes. Their horizontal axes represent the filtration value. If we rearrange the bars according to the node indices, and connect them following how new connections were introduced in the Rips filtration, the barcode is transformed to SLD.

complex and its element is a simplex [29]. A node is a 0-simplex, an edge is a 1-simplex, and a triangle is a 2-simplex. A complete graph with $p$ nodes represents the edges of a $(p-1)$-simplex.

Definition 3: A simplicial complex $\mathcal{K}$ is a finite collection of simplices such that [29]

1) any face of $\sigma \in \mathcal{K}$ is also in $\mathcal{K}$, and

2) for $\sigma_{1}, \sigma_{2} \in \mathcal{K}, \sigma_{1} \cap \sigma_{2}$ is a face of both $\sigma_{1}$ and $\sigma_{2}$.

The binary network $\mathcal{B}(X, \epsilon)$ is a simplicial complex consisting of 0 -simplices (nodes) and 1-simplices (edges) [43]. There are various simplicial complexes. One of them is the Rips complex.

Definition 4: Given a point cloud data $X$, the Rips complex $\mathcal{R}(X, \epsilon)$ is a simplicial complex whose $k$-simplices correspond to unordered $(k+1)$-tuples of points which are pairwise within distance $\epsilon$ [38]. 
While the binary network $\mathcal{B}(X, \epsilon)$ has at most 1-simplices, the Rips complex has at most $(p-1)$ simplices. So the Rips complex can have faces as well (Fig. 1). Trivially we always have $\mathcal{B}(X, \epsilon) \subseteq$ $\mathcal{R}(X, \epsilon)$ assuming we use the same metric in constructing the binary network and the Rips complex.

\section{B. Multiscale network as graph filtration}

So far we treated the network $\mathcal{B}(X, \epsilon)$ at a fixed threshold $\epsilon$ as a simplicial complex. When we change the threshold, we obtain a sequence of networks

$$
\mathcal{B}\left(X, \epsilon_{0}\right), \mathcal{B}\left(X, \epsilon_{1}\right), \mathcal{B}\left(X, \epsilon_{2}\right), \cdots
$$

We will explore the relationship among these networks.

When $\epsilon$ increases, the subsequent Rips complex becomes larger than all the previous Rips complex. Therefore, we have

$$
\mathcal{R}\left(X, \epsilon_{0}\right) \subseteq \mathcal{R}\left(X, \epsilon_{1}\right) \subseteq \cdots \subseteq \mathcal{R}\left(X, \epsilon_{n}\right)
$$

for $\epsilon_{0} \leq \epsilon_{1} \leq \cdots \leq \epsilon_{n}$. The nested sequence of the Rips complexes is called a Rips filtration, which is the main theme of persistent homology [29]. Similarly, we also have graph filtration for the case of the sequence of nested binary networks [44]

$$
\mathcal{B}\left(X, \epsilon_{0}\right) \subseteq \mathcal{B}\left(X, \epsilon_{1}\right) \subseteq \cdots \subseteq \mathcal{B}\left(X, \epsilon_{n}\right)
$$

for $\epsilon_{0} \leq \epsilon_{1} \leq \cdots \leq \epsilon_{n}$. This is the reason why we introduce the Rips complex in the first place. We need the basic mathematics of Rips complex in building graph filtration, which is a subset of Rips filtration.

As illustrated in Fig. 2 (b), as the filtration value $\epsilon$ changes, the topological characteristic of the Rips complex changes. The topological change of the filtration can be visualized using the barcode, which is constructed by plotting the changing topological features over different filtration values. The topological feature is displayed using a bar which starts and ends when the feature appears and disappears. The barcode represents the changes in topological features when the filtration value changes.

Among the many topological features, the zeroth Betti number $\beta_{0}$ counts the number of connected components in a network. We formally define the connected component in networks and simplicial complexes.

Definition 5: In a simplicial complex, a path between the two nodes is a sequence of nodes such that from each of its nodes there is an edge to the next node in the sequence. The connected component in the simplicial complex is a subset of which any two nodes are connected to each other by paths.

Since the $p$-th Betti number is estimated by the $p$ - and $(p+1)$-simplices, the binary network $B(X, \epsilon)$ contains enough information to compute $\beta_{0}$ [29]. In Fig. 2 (f), we plotted the zeroth Betti number $\beta_{0}$ (vertical) of the Rips complex over the filtration value (horizontal) [38]. The barcode is basically a decreasing function showing when the connected components are merging to form a bigger component.

The change of $\beta_{0}$ shows the topological change of a network before all nodes are connected. Until now, many brain network studies have concerned about the network properties after all nodes are connected. It is because they have focused on finding the small-worldness, scale-freeness or modularity. In this paper, 
we are interested in how the nodes are connected before all nodes are connected by observing the change of $\beta_{0}$.

\section{Multiscale network as dendrogram}

The barcode represents the global topological changes of a network visually; however, it lacks the geometric information of where the changes occur. That is the limitation of the barcode representation. By including an additional geometric information of node positions in the barcode, it is possible to transform the barcode into a dendrogram which provides a richer visual representation of how a brain network changes.

Consider the Rips filtration $\left\{\mathcal{R}\left(X, \epsilon_{k}\right) \mid k=0,1, \cdots\right\}$. Let $S_{m}^{k}$ and $S_{n}^{k}$ be the two disconnected components of the Rips complex $\mathcal{R}\left(X, \epsilon_{k}\right)$. Suppose that there are two nodes $x_{i} \in S_{m}^{k}$ and $x_{j} \in S_{n}^{k}$ such that the distance $d$ between $x_{i}$ and $x_{j}$ is less than the next filtration value $\epsilon_{k+1}$, i.e. $d\left(x_{i}, x_{j}\right)<\epsilon_{k+1}$. Then $S_{m}^{k}$ and $S_{n}^{k}$ will be connected at $\epsilon_{k+1}$. In other words, the components $S_{m}^{k}$ and $S_{n}^{k}$ will be connected at $\epsilon_{k+1}$ if

$$
d\left(S_{m}^{k}, S_{n}^{k}\right)=\min _{x_{i} \in S_{m}^{k}, x_{j} \in S_{n}^{k}} d\left(x_{i}, x_{j}\right)<\epsilon_{k+1} .
$$

Note that $d\left(S_{m}^{k}, S_{n}^{k}\right)$ is the single linkage distance between the clusters $S_{m}^{k}$ and $S_{n}^{k}$ often used in hierarchical clustering [40], [41]. Hence, the sequence of how components are merged during the Rips filtration is identical to the sequence of the merging in the dendrogram construction. To emphasize our main finding, we write it as a proposition.

Proposition 1: The sequence of how connected components are merged during the Rips filtration is identical to the sequence of the clustering in the single linkage dendrogram. The filtration value $\epsilon_{k+1}$ at which the two connected components $S_{m}^{k}$ and $S_{n}^{k}$ are merged is determined by the single linkage distance $d\left(S_{m}^{k}, S_{n}^{k}\right)$.

Fig. 2 (f) and (g) show the schematic of the relationship between the Rips filtration and the SLD construction. We used $d\left(x_{i}, x_{j}\right)=c_{X}\left(x_{i}, x_{j}\right)$ as the single linkage distance between the nodes. At each filtration value, the connected components are identified by the circles with different colors in (b). If we rearrange the bars in the barcode and connect the bars according to the node indexing and the Rips filtration, we obtain SLD (see the color of lines in (f-g)).

The barcode shows the global topological characteristics of when the components are merged while SLD shows the local network characteristics of what subnetworks are clustered together. Note that SLD is invariant under the permutation on node indices. Regardless of which nodes we start building SLD, our framework can always produce the consistent SLD. This is a useful property for a data set with extremely large number of nodes. The single linkage clustering is the only method that satisfies the uniqueness theorem for clustering algorithms and it is sufficiently stable for small perturbations in the metric [40], [41], [45].

\section{Single linkage matrix}

Given the network $\left(X, c_{X}\right)$, we were able to construct SLD. Then using SLD, we can recompute the distance between the nodes in the network using the single linkage distance $d_{X}$. We can view the original 
distance $c_{X}$ as the observed distance while the single linkage distance $d_{X}$ as the model predicted distance using SLD. Let $\left\{x_{i}=w_{0}, \cdots, w_{k}=x_{j}\right\}$ be a path between $x_{i}$ and $x_{j}$. Then the single linkage distance $d_{X}$ is formally given as

$$
\begin{aligned}
& d_{X}\left(x_{i}, x_{j}\right)= \\
& \min \left\{\max _{l=0, \cdots, k-1} c_{X}\left(w_{l}, w_{l+1}\right) \mid x_{i}=w_{0}, \cdots, w_{k}=x_{j}\right\} .
\end{aligned}
$$

The minimum is taken over every possible path between $x_{i}$ and $x_{j}$. For simplicity, $d_{X}=\left[d_{X}\left(x_{i}, x_{j}\right)\right]_{\forall i, j}$ is called as a single linkage matrix (SLM). Fig. 2 (e) shows the SLM $d_{X}$, which can be decomposed into the sequence of matrices representing the connected components in (d).

SLD can be easily understood through information diffusion during the filtration. Suppose that each node has its own information. At the filtration value $\epsilon=0$, the information starts to diffuse simultaneously from each node over the network. If the information meets by connecting edges, the information is mixed and the nodes belonging to the same connected component will share the mixed information. In this way, the single linkage distance between two nodes can be thought as the smallest diffusion rate to mix the information starting from the two nodes. The single linkage hierarchical clustering (dendrogram) visualizes this diffusion process by a tree diagram that depends on the filtration value.

\section{Distance BETWEEN NETWORKS}

Traditionally, the network comparison is performed by determining the difference between the graph theoretic measures such as assortativity, betweenness centrality, small-worldness and network homogeneity [12], [46], [47]. In persistent homology, there are various metrics that have been proposed to measure the distance between the two topological spaces. Probably the most widely used metric is the bottleneck distance that is often used in measuring the distance between the two persistence diagrams [48]. GH distance is also proposed to measure the distance between dendrograms [40], [41].

\section{A. Graph theoretic measures}

In this study, we considered seven most widely used graph measures: assortativity, betweenness centrality, clustering coefficient, characteristic path length, small-worldness, modularity and network homogeneity [49], [50], [51], [52], [47]. Other several network similarity measures such as the vertex similarity, graphlet degree distribution or P-Rank were not included [53], [54], [55]. While the first seven measures are defined in the both weighted and binary networks, the last three measures are only defined in the binary network. To compare the performance under the same condition, we only used the graph measures defined in the weighted network. Here we briefly explain the graph measures for completeness.

Assortativity is the correlation between the degrees of connected nodes [49]. Betweenness centrality is the average of the ratio of all shortest paths which pass through each node [50]. The clustering coefficient is the average of the fraction of triangles around each node and the characteristic path length is the average of the shortest path length between each pairwise nodes [51]. The fraction of the average clustering coefficient over the characteristic path length defines the small-worldness [51]. The modularity measures 
how the network can be subdivided into modules or communities [52]. When the number of edges within a module is larger while the number of edges between modules is smaller, the modularity becomes higher. Each graph measure reflects a different topological characteristic of the brain network. For example, the clustering coefficient and characteristic path length are related to the small-worldness. The assortativity and betweenness are related to the scale-freeness. The modularity is related to the community structure [56], [46]. The network homogeneity is a node-wise measure obtained by the mean correlation of the any given node with every other node within a given network [47].

Except for the characteristic path length, small-worldness and modularity, the other measures are defined in each node or edge in the network. Since we need a single scalar value representing the network for the comparison, we estimated the average assortativity, betweenness centrality and clustering coefficient. The global network homogeneity was obtained by calculating the sum of the network homogeneity scores [47].

Each graph measure is an algebraic invariant representing its own topological characteristic of the network. Using their Euclidean distances, we know whether the networks have similar topological properties or not.

\section{B. Bottleneck distance}

The bottleneck distance was originally defined for measuring the distance between two sets in the same metric space.

Definition 6: Let $X, Y$ be point cloud sets in the metric space $(Z, d)$. Each element of $X$ is paired with at least one element of $Y$. The bottleneck distance $d_{B}$ between $X$ and $Y$ is then given by

$$
d_{B}(X, Y)=\min _{f: X \rightarrow Y} \max _{x \in X}|x-f(x)|
$$

If we plot the birth and death time of a chosen topological feature over the filtration in the horizontal and vertical axes respectively, we obtain the persistence diagram [29]. The bottleneck distance is applied to measure the difference between two persistence diagrams.

In this study, we are interested in how the number of the connected components as the main topological feature of interest. In the graph filtration, the zeroth Betti number $\beta_{0}$ always decreases and no new component is born. Hence the birth time is always fixed at the filtration value 0 and only the death time is varying. Since the number of nodes is fixed for all brain networks, the number of connected components is identical in each network. Then, the one-to-one function $f$ from $X$ to $Y$ is simply determined by the death time. The bottleneck distance for the brain network is the maximum difference between two sequences of the ordered single linkage distances, i.e., filtration values when the two disjoint components are connected.

\section{Gromov-Hausdorff distance}

The Hausdorff and bottleneck distances are usually defined for different point sets in the same metric space. However, this is not a useful metric in measuring the distance between networks because each 

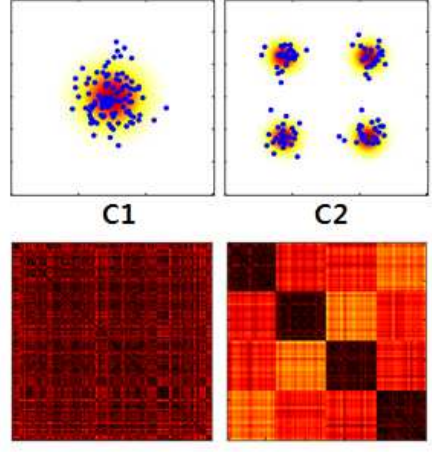

C2
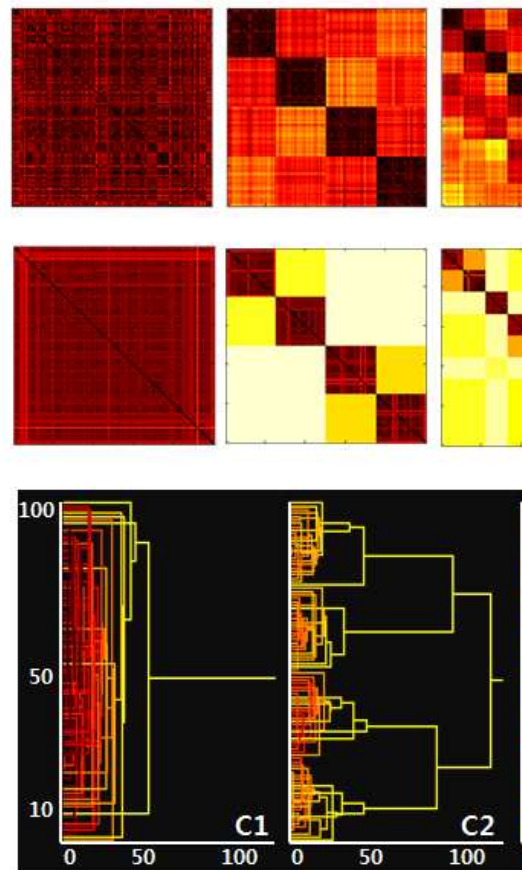

100

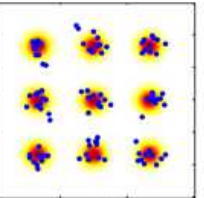

C3 (a)

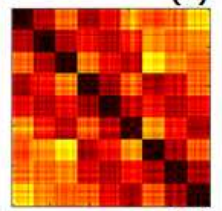

(b)

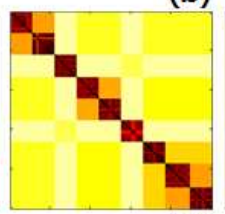

(c)

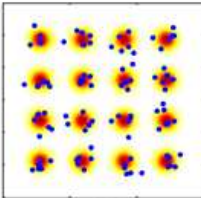

C4
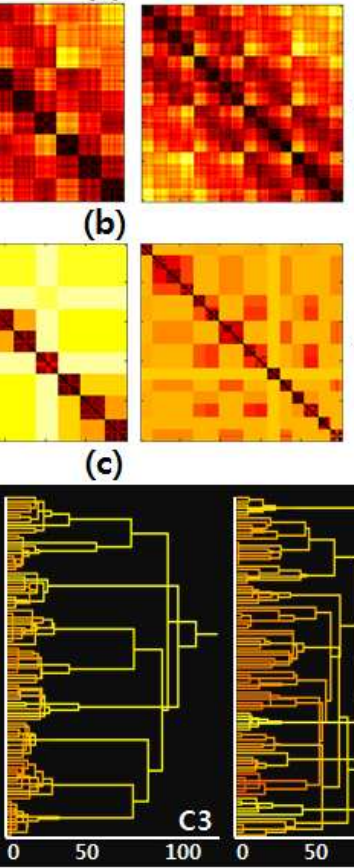

(d)
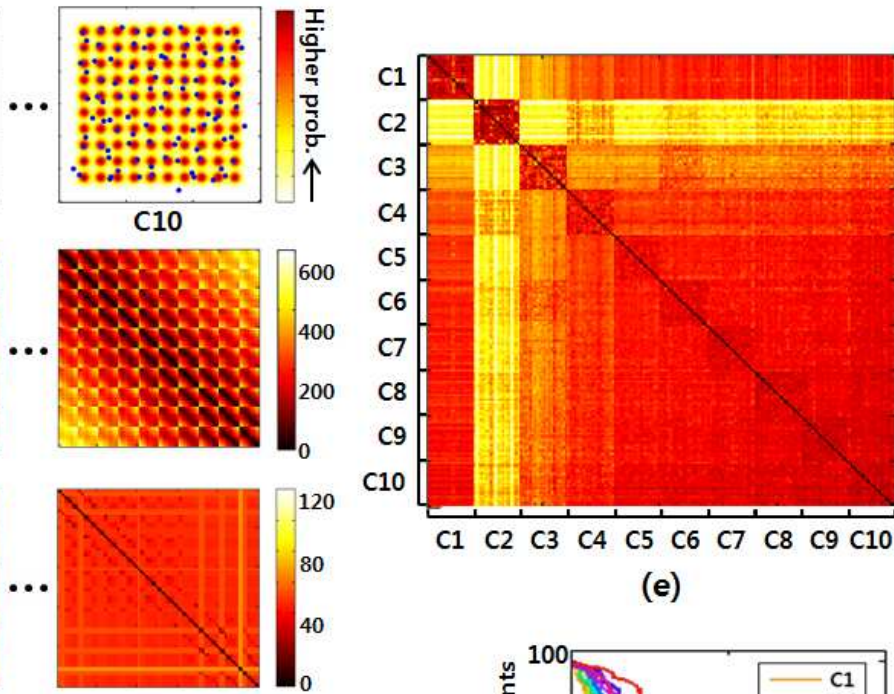

(e)

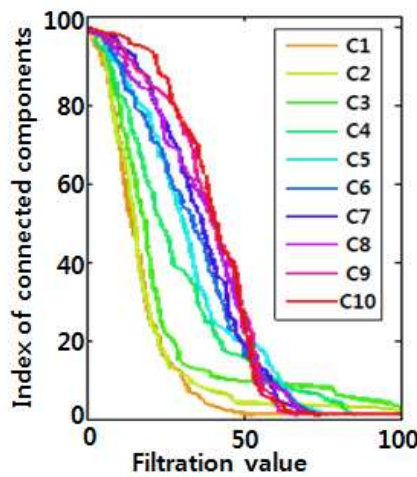

(f)

Fig. 3. Simulated results. (a) 10 probability maps for sampling the data points. Each map is composed of $1,4,9, \ldots 100$ bivariate normal distributions from left to right and they are denoted as $\mathrm{C} 1, \mathrm{C} 2, \ldots, \mathrm{C} 10$, respectively. We sampled 100 data points from each probability map like the blue dots in each panel. (b) The distance matrices, (c) SLMs, (d) SLDs and (f) barcodes of $\beta_{0}$ of the sampled datasets. In (d) and (e), the horizontal axes represent the filtration value and the vertical axes represent the index of connected component and one of nodes, respectively. The slope of barcodes become more steep going from $\mathrm{C} 1$ to C10. 20 datasets with 100 datapoints are generated from each probability map. The total number of datasets is $20 \times 10=200$. We computed their SLMs using $20 \times 10$ datasets and GH distances of all pairwise SLMs. Then, $200 \times 200 \mathrm{GH}$ distance matrix are obtained as shown in (e). When we clustered 200 SLMs using Ward's cluster analysis, the clustering accuracy was $82.5 \%$.

network will have its own metric. So what we need is a new metric, GH distance, that can be used to measure the distance between different metric spaces [57]. In computing the GH distance, we need to determine the correspondence between two different metric spaces, $X$ and $Y$. In our brain network model, the node sets $X$ and $Y$ is given in the fixed identical locations in the template. Therefore, the node $x_{i} \in X$ is simply mapped to $y_{i} \in Y$ [44], [57]. Therefore, GH-distance can be trivially discretized as

$$
d_{G H}(X, Y)=\frac{1}{2} \max _{x_{i} \in X, y_{j} \in Y}\left|d_{X}\left(x_{i}, x_{j}\right)-d_{Y}\left(y_{i}, y_{j}\right)\right| .
$$

The GH distance is the maximum difference between two SLMs when the order of column and row vector is fixed. While the bottleneck distance finds when the difference is maximized between two networks during the changing of the number of connected components, the GH distance finds where the difference is maximized between networks. 


\section{ApPlicAtion to Simulation DATA}

We applied the proposed method to the simulation data shown in Fig. 3 (a). For replicating our results, we have provided the simulation data and MATLAB codes at http://sites.google.com/site/hkleebrain/home/persistent-homology-2. We used 10 probability maps which are composed of $1,4,9,16, \ldots, 100$ bivariate normal distributions. We denoted the probability maps from left to right panels as $\mathrm{C} 1, \mathrm{C} 2, \ldots, \mathrm{C} 10.100$ data points are sampled from each probability map (blue dots). Each data point is considered as a node and the Euclidean distances is used for edge weights. Then, their distance matrices, SLMs, SLDs and barcodes of $\beta_{0}$ are computed and shown respectively in (b), (c), (d) and (f). The distance matrices and SLMs are in $\mathbb{R}^{100 \times 100}$. In the dendrogram (d), the horizontal and vertical lines represent the connected components and the merging of two connected components. Its color is varied according to the distance to the giant component, which is a connected component when all nodes are connected. We set the distance from giant component to giant component as one. Whenever the component is divided into two smaller components, the distance increases one by one. The colorbar for the distance to the giant component is shown in the left. We illustrated the barcode of $\beta_{0}$ as the decreasing function in (f). The color of each line is varied according to the corresponding datasets, $\mathrm{C} 1, \ldots, \mathrm{C} 10$. When the dataset is changed from $\mathrm{C} 1$ to $\mathrm{C} 10$, the barcode has flatter peak, lighter tail and steeper slope. We generated 200 datasets by sampling 20 datasets per probability map. We estimated the distance between all pairwise SLMs using GH metric. The obtained $200 \times 200$-dimensional GH distance matrix is shown in (e). Using GH distance matrix, we clustered 200 SLMs into 10 clusters based on Ward's linkage cluster analysis. During the clustering, we assumed that the group labels of all SLMs are unknown. After clustering, the clustered labels are compared to the true labels. The clustering accuracy is $82.5 \%$. Most of the mis-clustering occurred in C9 and C10.

\section{Application to Brain Network Model}

In this paper, we applied the proposed framework in constructing functional brain networks with 103 ROIs extracted from FDG-PET data. FDG-PET measures glucose metabolism, which is associated with neuronal activity [58]. The interregional metabolic correlation between brain regions was used to reflect functional connectivity during the resting state [59], [60], [61]. While the resting state fMRI records the blood oxygenation level dependent (BOLD) signal every 2 or 3 seconds, FDG-PET records the FDG uptake for 30 minutes after 20 minutes from the injection. Thus, FDG-PET data at the resting state is more stationary and invariant to the noise compared to fMRI studies.

\section{A. Subjects}

FDG-PET was scanned from three groups. They were recruited from Child and Adolescent Psychiatric Outpatient Clinic of Seoul National University Hospital, Seoul, South Korea. Twenty-four children with ADHD (19 boys and 5 girls, mean age: $8.2 \pm 1.6$ years) were examined. They were diagnosed by a board certified child and adolescent psychiatrist using DSM-IV diagnostic criteria, Korean version of ADHD rating scale IV (K-ARS) and, Korean version of Kiddie-Schedule for Affective Disorders and 
Schizophrenia-Present and Lifetime version (K- SADS-PL). Twenty-six children with ASD (24 boys and 2 girls, mean age: $6.0 \pm 1.8$ years). They were diagnosed by the Korean version of the Autism Diagnostic Interview-Revised (K-ADI-R) and the Korean version of the Autism Diagnostic Observation Schedule (ADOS). The pediatric controls comprised 11 children (7 boys and 4 girls, mean age: $9.7 \pm 2.5$ years). They visited our clinic but failed to meet the criteria of any psychiatric disorder or visited a Child and Adolescent Psychiatric Outpatient Clinic of Seoul National University Hospital for IQ evaluation only. This study was approved by the Institutional Review Board of Seoul National University College of Medicine.

\section{B. PET image acquisition and preprocessing}

All PET scans were obtained using an ECAT EXACT 47 (Siemens-CTI, Knoxville, USA) PET scanner with an intrinsic resolution of $5.2 \mathrm{~mm}$ FWHM. An emission scan was obtained with FDG dose of 0.3 $\mathrm{mCi} / \mathrm{kg}$ for 30 minutes during resting state, after a transmission scan measured by $68 \mathrm{Ge}$ rod sources for attenuation correction. All participants were scanned under normal environmental noise of the scanner room. A filtered back-projection algorithm (Shepp-Logan filter at a cutoff frequency of 0.3 cycles/pixel as $128 \times 128 \times 47$ matrices of size $2.1 \times 2.1 \times 3.4 \mathrm{~mm}$ ) was used for transaxial image reconstruction. PET images were spatially normalized to the Korean standard template space after converting to Analyze format and smoothed with a Gaussian filter of $16 \mathrm{~mm}$ FWHM using Statistical Parametric Mapping (SPM 2, University College of London, UK), implemented in the Matlab 6.5 (Mathworks Inc., USA) environment [62]. To minimize the any ethnic differences, we used Korean standard PET and MR templates developed using the normal Korean volunteers [62]. The procedure for obtaining the FDG-values from 103 ROIs is as follows:

1) The ROIs were manually parcellated in high resolution MRI and the weighted probabilities of ROIs were estimated based on the population.

2) The ROIs drown in MR image were warped into Korean PET template registered to Korean MR template, which is called as Korean statistical probabilistic anatomical map (KSPAM). It provides effective tools for quantifying the regional intensity of FDG uptake of PET data. In this way, we have probabilistic ROIs in PET template space.

3) All PET images were then normalized to PET template using the affine transformation.

4) The FDG-value of ROI in PET template space were extracted by the weighted averaging with the weights given by the weighted probability.

The value of FDG uptake was globally normalized to the individuals total gray matter mean count. This normalized value was obtained from 103 ROIs as follows: 20 frontal, 20 parietal, 30 temporal, 16 subcortical, 14 occipital and 3 cerebellar regions. The age effect was factored out from 103 ROIs using a general linear model.

\section{Results}

Using the predefined 103 ROIs, we constructed the brain networks following the proposed framework. We will also validate our proposed framework with more available graph theoretic approaches. 


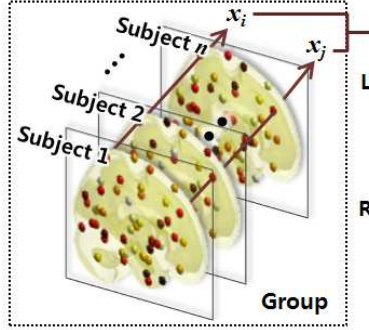

(a)

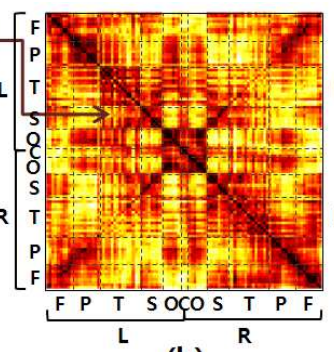

(b)

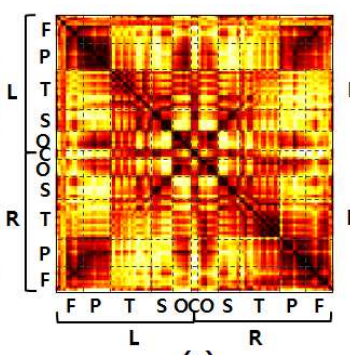

(c)

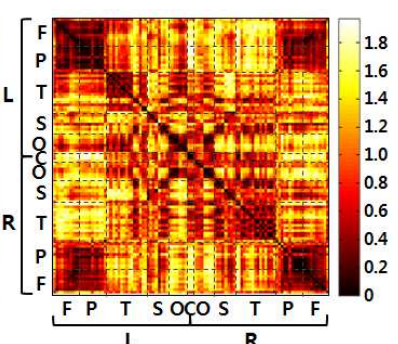

(d)

Fig. 4. (a) Procedure for constructing the correlation-based distance map $c_{X}$ (1) using the given dataset. (b-d) The obtained distance maps for ADHD, ASD and PedCon groups. F, P, T, S, O and C represent frontal, parietal, temporal, subcortical, occipital and cerebellar regions. $\mathrm{L}$ and $\mathrm{R}$ indicate the left and right hemispheres.

\section{A. Multiscale brain networks}

We computed the correlation-based distance $c_{X}$ (1) for ADHD, ASD and PedCon groups in Fig. 4. The multiscale brain networks were constructed using the graph filtrations on ADHD, ASD and PedCon populations.

The distance metric we used is 1-correlation. So the distance is always between 0 and 2 . The negative correlation corresponds to the distance larger than 1. However, during the filtration, almost all nodes are connected before epsilon value reaches 1 in our data set without exception. The nodes merge together quickly in the filtration to form a single giant connected component. So we did not really consider negative correlations and the filtration is done between 0 and 1 .

The graph filtrations at 8 different values $\epsilon=0.05,0.1,0.15, \ldots, 0.35$ are shown in Fig. 5 (a) ADHD, (b) ASD and (c) PedCon. The color of node is changed according to the connected component to which the node belongs. At $\epsilon=0$, the color of nodes (connected components) is shown in the colorbar. When $\epsilon$ increases, the color of node is changed. We also illustrated the barcodes for the three groups in (d). The maximum single linkage distances of ADHD, ASD and PedCon are 0.40,0.51, and 0.47.

In the barcode for $\beta_{0}$, the decreasing slopes are 364,300, and 314 for ADHD, ASD and PedCon. The single linkage distance matrices for ADHD, ASD and PedCon were also computed in Fig. 6 (a-c). The single linkage dendrograms within a network were illustrated in Fig. 6 (d-f). The color of lines in the dendrogram represents the distance to the giant component for each connected component. The maximum distances to the giant component are 25,31 and 26 for ADHD, ASD and PedCon, respectively.

\section{B. Performance against other network measures}

The 3D static PET image is obtained one per subject. Since we do not have multiple PET scans per subject, we couldn't generate a single network per subject. So we used the Jackknife resampling technique to generate additional networks. For a group with $n$ subjects, one subject is removed and the remaining $n-1$ subjects are used to construct a network. This process is repeated for each subject to produce $n$ networks for the group. Therefore, we were able to simulate 24, 26 and 11 networks for ADHD, ASD and PedCon groups. For all $(24+26+11)$ networks, we estimated the SLM, barcode of $\beta_{0}$, slope of barcode 


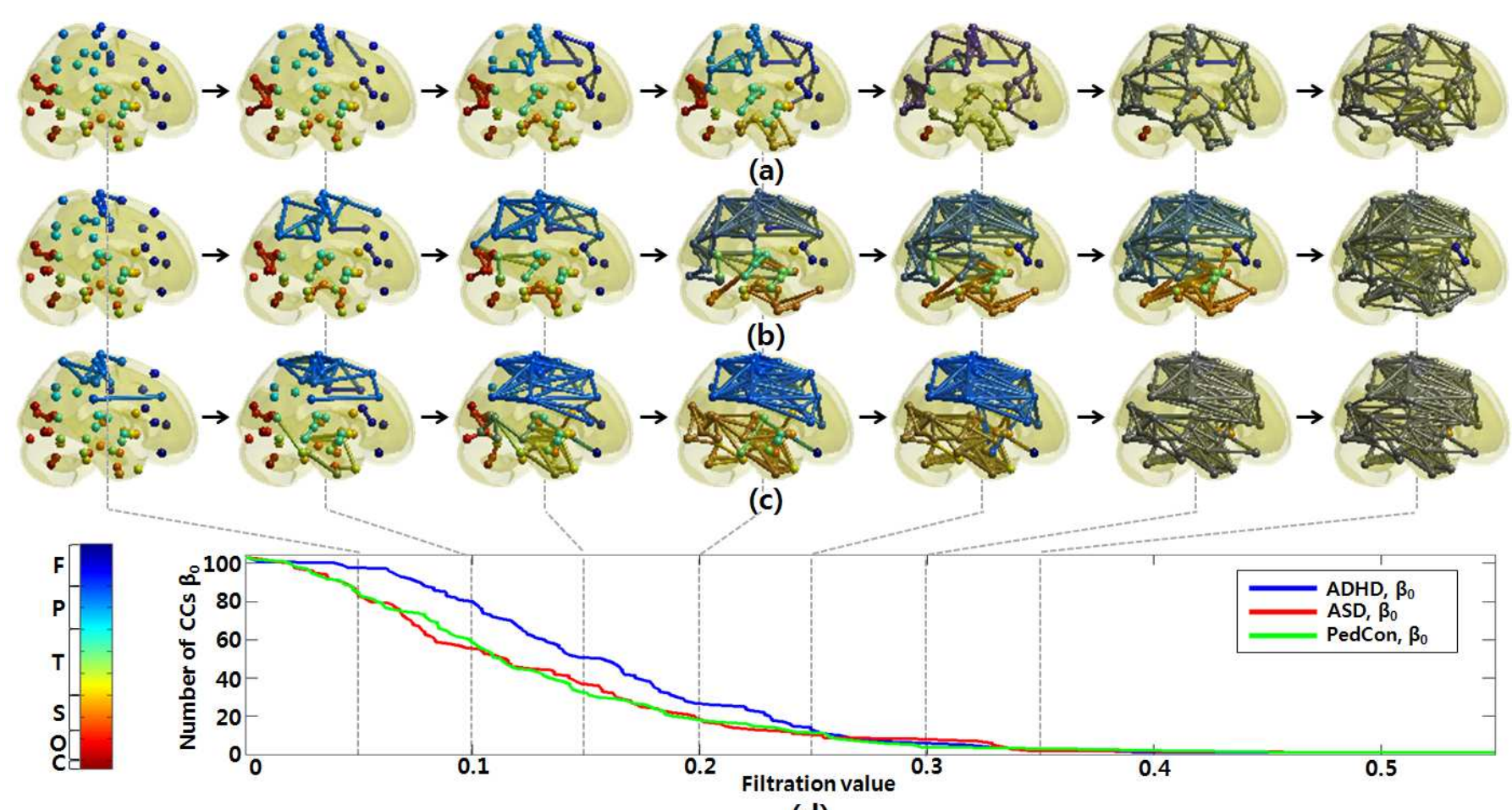

(d)

Fig. 5. Graph filtration of (a) ADHD, (b) ASD and (c) PedCon at the filtration values $\epsilon=0.1,0.15,0.2, \ldots, 0.45$. The color of nodes at $\epsilon=0$ is shown in the colorbar. If the nodes belong to the same connected component, they are colored identically. The barcodes $\beta_{0}$ of ADHD, ASD and PedCon are shown in (d).

$\beta_{0}$, average assortativity, average node betweenness centrality, average clustering coefficient, characteristic path length, small-worldness, modularity and the global network homogeneity. Then, the GH distance was computed between all pairwise SLMs. The bottleneck distance was computed similarly for all barcodes. For the other 8 graph theoretic measures, all pairwise differences were also computed. We obtained total 10 distance matrices between the networks. The result is summarized in Fig. 7, where each distance matrix is normalized with the maximum value 1 . The size of the distance matrices is $(24+26+11) \times(24+26+11)$, which corresponds to the number of simulated networks in the three groups. We clustered the simulated networks into 3 groups using the Ward's cluster analysis using each computed distance matrix. During the clustering we assume that the true group labels are unknown. Then, we evaluated the clustering accuracy by comparing the assigned labels with the true labels. The obtained clustering accuracies are 100\%, 44.26\%, $86.89 \%, 80.33 \%, 85.25 \%, 80.33 \%, 96.72 \%, 93.44 \%, 60.66 \%$ and $96.72 \%$ for GH distance, bottleneck distance, slope of barcode $\beta_{0}$, assortativity, betweenness centrality, clustering coefficient, characteristic path length, small-worldness, modularity and global network homogeneity, respectively.

We conclude that the GH-distance based approach shows the best performance against 10 available methods. Surprisingly the bottleneck distance predominantly used in persistence homology performed poorly possibly due to the fact that it ignored the geometric information of network nodes. 


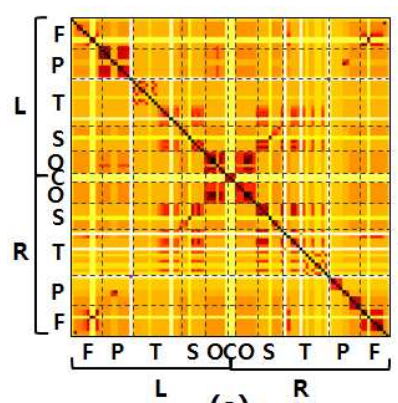

(a)

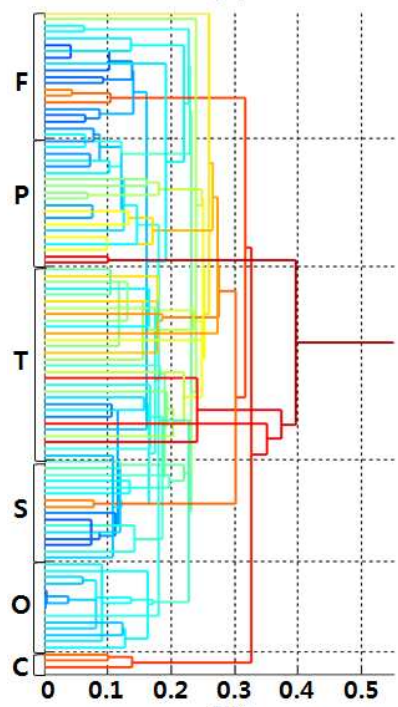

(d)

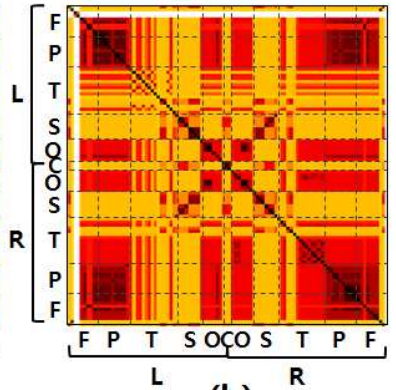

(b)

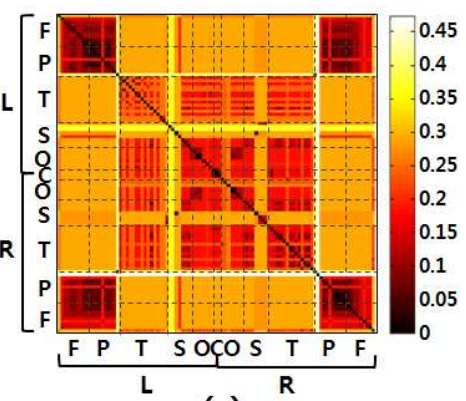

(c)

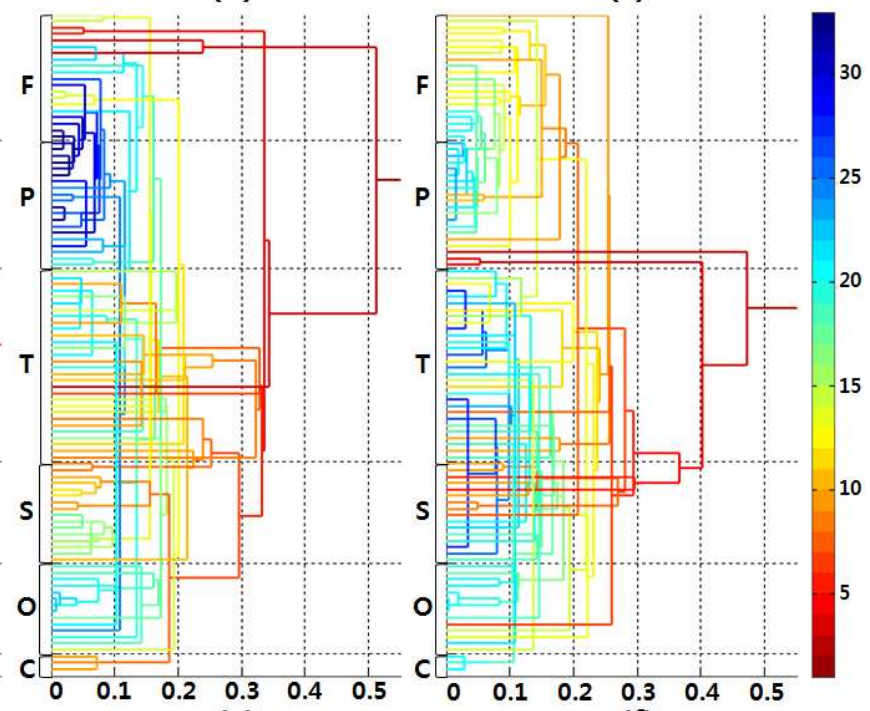

(e)

Fig. 6. (a-c) SLMs $d_{X}$ of ADHD, ASD and PedCon obtained from the original correlation-based distance $c_{X}$ in Fig. 4 (b-d). Compared to the correlation-based distance $c_{X}$, we have better group separation in the single linkage distance $d_{X} . \mathrm{F}, \mathrm{P}, \mathrm{T}, \mathrm{S}, \mathrm{O}$ and $\mathrm{C}$ represent frontal, parietal, temporal, subcortical, occipital and cerebellar regions. L and R indicate the left and right hemispheres. (d-f) Single linkage dendrograms of ADHD, ASD and PedCon. The vertical and horizontal axes represent the node index and filtration value. The color of lines shows the distance to the giant component. The distance to the giant component of the giant component is 1 . Whenever the connected component is divided into the smaller components, the distance increases one by one.

\section{DISCUSSION}

\section{A. Summary}

In this paper, we proposed a new framework for modeling brain connectivity using the persistent homology. The proposed framework avoids the traditional thresholding of connectivity matrices in obtaining binary networks. Instead of trying to find an optimal threshold, which may not be optimal for other networks, we constructed networks for every possible threshold. Then we determined the persistent topological features over the evolution of the network changes at every scale.

In developing a unified mathematical framework, we borrowed heavily from the persistent homology. In this paper, we showed that binary networks are the subsets of the Rips complex, and the collection of the networks over different scales forms the Rips filtration. For the visualization of the multiscale networks, we used the barcode and the dendrogram, and explored the relationship between the two representations.

For quantification, we used the single linkage distance and GH-distance. We showed that the GH- 


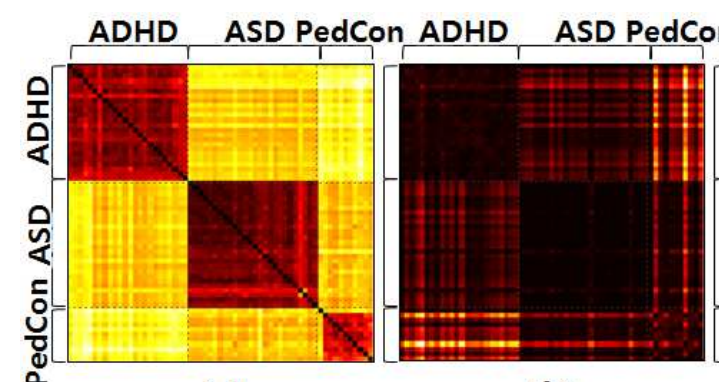

(a) (b)

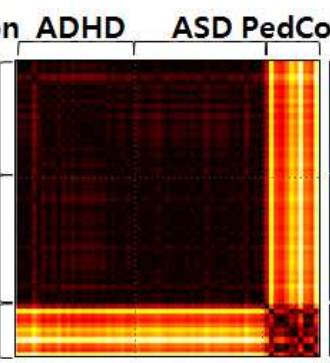

(c)

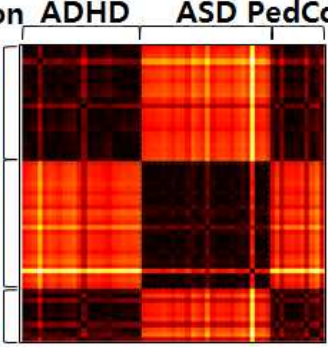

(d)

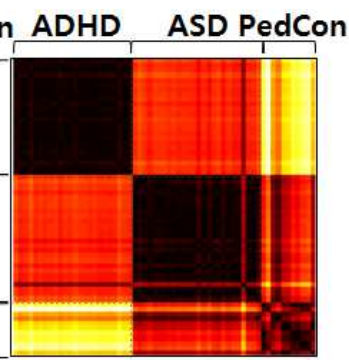

(e)

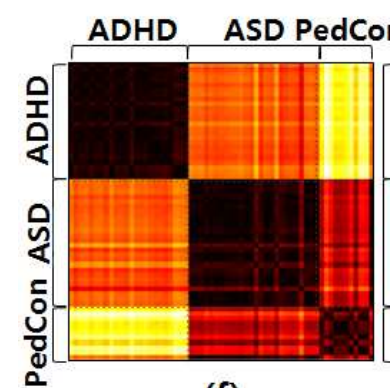

(f)

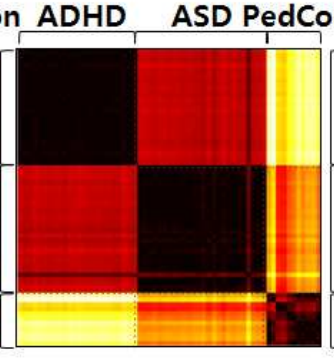

(g)

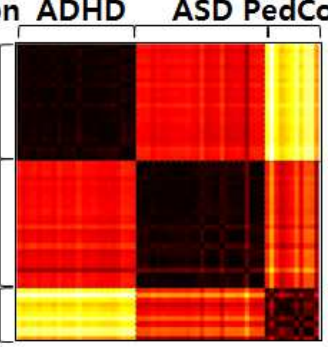

(h)

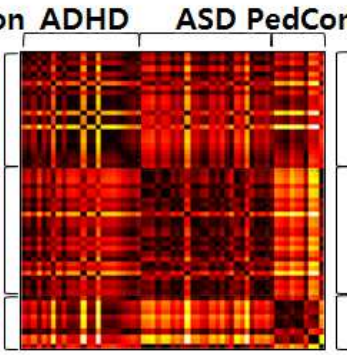

(i)

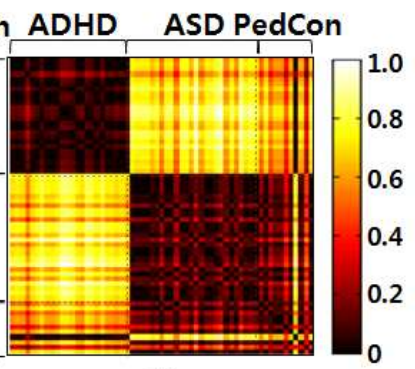

(j)

Fig. 7. Comparison of 11 different methods: (a) GH distance, (b) bottleneck distance, (c) slope of barcode $\beta_{0}$, (d) assortativity, (e) betweenness centrality, (f) clustering coefficient, (g) characteristic path length, (h) small-worldness, (i) modularity and (j) global network homogeneity. In each distance matrix, the 3 diagonal block matrices with the size $24 \times 24,26 \times 26$ and $11 \times 11$ measure the distance between networks within a group and the off-diagonal block matrices measure the distance between groups. The clustering accuracy using the Ward's cluster method was also performed. From (a) to (j), the clustering accuracies are 100\%, 44.26\%, 86.89\%, 80.33\%, 85.25\%, $80.33 \%, 96.72 \%, 93.44 \%, 60.66 \%$ and $96.72 \%$. The GH-distance shows the best performance.

distance has better discriminating power than the bottleneck distance and many other graph theoretic measures.

\section{B. Network findings}

In Fig. $5(\mathrm{~d})$, the slopes of barcode $\beta_{0}$ are ASD $<$ PedCon $<$ ADHD with the significance level .05 based on the Wilcoxon rank-sum test using the resampled datasets. The filtration values when all nodes are connected is generated are ADHD $\approx$ PedCon $<$ ASD with the significance level .05. It implies that ASD has longer heavy tail in the shape of barcode (globally disconnected until the larger filtration values) and sharper peak (locally connected at the smaller filtration values) compared to PedCon and ADHD. The sharp peak and long heavy tail of ASD is related to local over-connectivity and global under-connectivity, respectively [63].

For pairwise comparisons of the single linkage distances (Fig. 6), the Wilcoxon rank-sum test was used with Bonferroni's correction. We found that ASD showed loosely connected between left inferior prefrontal regions such as BA44 and BA45 and other brain regions (corrected $p<.05$ ). The loose coupling of inferior frontal area 44/45 might be reflected the behavioral symptom in ASD [64]. We found that ADHD showed loosely connected pattern between sensorimotor region and various frontoparietal regions including anterior cingulate compared to PedCon (corrected $p<.05$ ). This loose connected pattern might reflect the deficits of cognitive attentional control [65] and sensori-motor integration [66]. Also, ASD and 
ADHD have commonly abnormal connected structure in cerebellum (corrected $p<.05$ ). Cerebellum is one of the pathophysiological regions in ADHD and ASD. In this way, the single linkage distance using network filtration could be an another indicator for characterizing abnormal brain network.

\section{Future works}

In this paper, we considered the graph filtration. and only the zeroth Betti number $\beta_{0}$ was used as the topological invariant. However, since the functional brain network itself is not in the 3-dimensional Euclidean space where the nodes are embedded but in a higher dimensional space, we also need to consider higher degree Betti numbers beyond the zeroth by extending the concept of network filtration to the Rips complex.

For a network with an extremely large number of nodes, the construction of the Rips filtration and the corresponding single linkage dendrogram is time consuming. Since the construction of a single linkage dendrogram is equivalent to that of the minimum spanning tree (MST) [44], it is possible to speed up the computation by using either the Prim's or Kruskal's algorithm that are often used in finding MST. The relationship between MST and our persistent homological framework is also left out for a future study.

\section{REFERENCES}

[1] S. Achard, R. Salvador, B. Whitcher, J. Suckling, and E. Bullmore, "A resilient, low-frequency, small-world human brain functional network with highly connected association cortical hubs," J. Neurosci., vol. 26, pp. 63-72, 2006.

[2] D. S. Bassett, "Small-world brain networks," Neuroscientist, vol. 12, pp. 512-523, 2006.

[3] Z. J. Chen, Y. He, P. Rosa-Neto, J. Germann, and A. C. Evans, "Revealing modular architecture of human brain structural networks by using cortical thickness from MRI," Cereb. Cortex, vol. 18, pp. 2374-2381, 2008.

[4] V. M. Eguiluz, D. R. Chialvo, G. A. Cecchi, M. Baliki, and A. V. Apkarian, "Scale-free brain functional networks," Phys. Rev. Lett., vol. 94, p. 018102, 2005.

[5] Y. He, Z. Chen, and A. Evans, "Small-world anatomical networks in the human brain revealed by cortical thickness from MRI," Cereb. Cortex, vol. 170, pp. 2407-2419, 2007.

[6] D. Meunier, S. Achard, A. Morcom, and E. Bullmore, "Age-related changes in modular organization of human brain functional networks," NeuroImage, vol. 44, pp. 715-723, 2009.

[7] O. Sporns and J. D. Zwi, “The small world of the cerebral cortex,” Neuroinformatics, vol. 2, pp. 145-162, 2004.

[8] C. J. Stam, "Functional connectivity patterns of human magnetoencephalographic recordings : a small-world network?" Neurosci. Lett., vol. 355 , pp. 25-28, 2004.

[9] M. P. Van den Heuvel, C. J. Stam, M. Boersma, and H. E. Hulshoff Pol, "Small-world and scale-free organization of voxel-based resting-state functional connectivity in the human brain," NeuroImage, vol. 43, pp. 528-539, 2008.

[10] C. Zhou, L. Zemanová, G. Zamora, C. C. Hilgetag, and J. Kurths, "Hierarchical organization unveiled by functional connectivity in complex brain networks," Phys. Rev. Lett., vol. 97, p. 238103, 2006.

[11] O. Sporns, G. Tononi, and R. Kotter, "The human connectome: a structural description of the human brain," PLoS Comput. Biol., vol. 1, p. e42, 2005.

[12] E. Bullmore and O. Sporns, "Complex brain networks: graph theoretical analysis of structural and functional systems," Nature Rev. Neurosci., vol. 10, pp. 186-198, 2009.

[13] K. J. Friston, L. Harrison, and W. Penny, "Dynamic causal modelling," NeuroImage, vol. 19, pp. 1273-1302, 2003.

[14] G. Marrelec, A. Krainik, H. Duffau, M. Pélégrini-Issac, S. Lehéricy, J. Doyon, and H. Benalia, "Partial correlation for functional brain interactivity investigation in functional MRI," NeuroImage, vol. 32, pp. 228-237, 2006.

[15] A. R. McIntosh and F. Gonzalez-Lima, "Structural equation modeling and its application to network analysis in functional brain imaging," Hum. Brain Mapp., vol. 2, pp. 2-22, 1994. 
[16] W. D. Penny, K. E. Stephan, A. Mechelli, and K. J. Friston, “Comparing dynamic causal models,” NeuroImage, vol. 22, pp. 1157-1172, 2004.

[17] O. Sporns, Networks of the Brain. The MIT Press, 2010.

[18] M. E. Newman, Networks: An Introduction. Oxford University Press, 2010.

[19] Y. He, Z. Chen, and A. Evans, "Structural insights into aberrant topological patterns of large-scale cortical networks in alzheimers disease," J. Neurosci., vol. 28, pp. 4756-4766, 2008.

[20] B. C. M. Wijk, C. J. Stam, and A. Daffertshofer, "Comparing brain networks of different size and connectivity density using graph theory," PLoS ONE, vol. 5, p. e13701, 2010.

[21] J. W. Bohland, H. Bokil, C. B. Allen, and P. P. Mitra, "The brain atlas concordance problem: Quantitative comparison of anatomical parcellations," PLOS ONE, vol. 4, p. e7200, 2009.

[22] L. Ferrarini, I. M. Veer, E. Baerends, M.-J. van Tol, R. J. Renken, N. J. van der Wee, D. J. Veltman, A. Aleman, F. G. Zitman, B. W. Penninx, M. A. van Buchem, J. H. Reiber, S. A. Rombouts, and J. Milles, "Hierarchical functional modularity in the resting-state human brain," Hum. Brain Mapp., vol. 30, pp. 2220-2231, 2009.

[23] M. Rubinov, S. A. Knock, C. J. Stam, S. Micheloyannis, A. W. Harris, L. M. Williams, and M. Breakspear, "Small-world properties of nonlinear brain activity in schizophrenia," Hum. Brain Mapp., vol. 30, pp. 403-416, 2009.

[24] R. Salvador, J. Suckling, M. R. Coleman, J. D. Pickard, D. Menon, and E. Bullmore, "Neurophysiological architecture of functional magnetic resonance images of human brain," Cereb. Cortex, vol. 15, pp. 1332-1342, 2005.

[25] S. Achard and E. Bullmore, "Efficiency and cost of economical brain functional networks," PLoS Comput. Biol., vol. 3, p. e17, 2007.

[26] M. A. Kramer, E. D. Kolaczyk, and H. E. Kirsch, "Emergent network topology at seizure onset in humans," Epilepsy Res., vol. 79, pp. 173-186, 2008.

[27] M. P. Van den Heuvel, C. J. Stam, R. S. Kahn, and H. E. Hulshoff Pol, "Efficiency of functional brain networks and intellectual performance," J. Neurosci., vol. 29, pp. 7619-7624, 2009.

[28] D. S. Bassett, A. Meyer-Lindenberg, S. Achard, T. Duke, and E. Bullmore, "Adaptive reconfiguration of fractal small-world human brain functional networks," P. Natl. Acad. Sci. USA, vol. 103, pp. 19518-19 523, 2006.

[29] H. Edelsbrunner and J. Harer, Computational Topology: An Introduction. American Mathematical Society Press, 2009.

[30] D. Horak, S. Maletić, and M. Rajković, "Persistent homology of complex networks," J. Stat. Mech-Theory E, vol. 2009, p. P03034, 2009.

[31] A. Zomorodian and G. Carlsson, "Computing persistent homology,” Discrete Comput. Geom., vol. 33, pp. 249-274, 2005.

[32] R. J. Adler, O. Bobrowski, M. S. Borman, E. Subag, and S. Weinberger, "Persistent homology for random fields and complexes," ArXiv e-prints, 2010.

[33] M. K. Chung, P. Bubenik, and P. T. Kim, "Persistence diagrams of cortical surface data," in IPMI '09: Proceedings of the 21st International Conference on Information Processing in Medical Imaging, 2009, pp. 386-397.

[34] G. Singh, F. Memoli, T. Ishkhanov, G. Sapiro, G. Carlsson, and D. L. Ringach, "Topological analysis of population activity in visual cortex," J. Vision, vol. 8, pp. 1-18, 2008.

[35] D. Pachauri, C. Hinrichs, M. Chung, S. Johnson, and V. Singh, "Topology based kernels with application to inference problems in alzheimer's disease," Medical Imaging, IEEE Transactions on, pp. 1760-1770, 2011.

[36] H. Lee, M. K. Chung, , H. Kang, B. N. Kim, and D. S. Lee, "Discriminative persistent homology of brain networks," in IEEE International Symposium on Biomedical Imaging (ISBI), Chicago, IL, 2011, pp. 841-844.

[37] G. Carlsson, A. Collins, and L. J. Guibas, "Persistence barcodes for shapes,” Int. J. Shape Model, vol. 11, pp. 149-187, 2005.

[38] R. Ghrist, "Barcodes: the persistent topology of data," B. Am. Math. Soc., vol. 45, pp. 61-75, 2008.

[39] T. Hastie, R. Tibshirani, and J. Friedman, The Elements of Statistical Learning. New York: Springer, 2009.

[40] G. Carlsson and F. Mémoli, "Persistent clustering and a theorem of J. Kleinberg," ArXiv e-prints, 2008.

[41] — "Characterization, stability and convergence of hierarchical clustering methods," J. Mach. Learn. Res., vol. 11, pp. 1425-1470, 2010.

[42] J. C. Hart, "Computational topology for shape modeling," in Proceedings of the International Conference on Shape Modeling and Applications, Washington, DC, USA, 1999, pp. 36-43. [Online]. Available: http://portal.acm.org/citation.cfm?id=829509.830259

[43] J. Erickson, "Computational topology notes," 2009. [Online]. Available: http://compgeom.cs.uiuc.edu/ jeffe/teaching/comptop/

[44] H. Lee, M. K. Chung, , H. Kang, B. N. Kim, and D. S. Lee, "Computing the shape of brain network using graph filtration and gromovhaudorff metric," in 14th International Conference on Medical Image Computing and Computer Assisted Intervention (MICCAI), vol. 6891, Toronto, Canada, 2011, pp. 289-296. 
[45] R. B. Zadeh and S. Ben-David, "A uniqueness theorem for clustering," in Proceedings of the International Conference on Uncertainty in Artificial Intelligence (UAI), Corvallis, Oregon, 2009, pp. 639-646.

[46] M. Rubinov and O. Sporns, "Complex network measures of brain connectivity: Uses and interpretations," NeuroImage, vol. 52, pp. 1059-1069, 2010. [Online]. Available: http://www.brain-connectivity-toolbox.net

[47] L. Q. Uddin, A. M. Kelly, B. B. Biswal, D. S. Margulies, Z. Shehzad, D. Shaw, M. Ghaffari, J. Rotrosen, L. A. Adler, F. X. Castellanos, and M. P. Milham, "Network homogeneity reveals decreased integrity of default-mode network in adhd," J. Neurosci., vol. 169, pp. 249-254, 2008.

[48] D. Cohen-Steiner, H. Edelsbrunner, and J. Harer, "Stability of persistence diagrams," Discrete Comput. Geom., vol. 37, pp. 103-120, 2007.

[49] M. E. Newman, "Assortative mixing in networks," Phys. Rev. Lett., vol. 89, p. 208701, 2002.

[50] L. Freeman, "Centrality in social networks: Conceptual clarification,” Soc. Networks, vol. 1, pp. 215-239, 1978.

[51] D. J. Watts and S. H. Strogatz, "Collective dynamics of small-world networks," Nature, vol. 393, pp. 440-442, 1998.

[52] M. E. Newman, "Fast algorithm for detecting community structure in networks," Phys. Rev. E, vol. 69, p. $066133,2004$.

[53] E. A. Leicht, P. Holme, and M. E. J. Newman, "Vertex similarity in networks," Phys. Rev. E, vol. 73, p. $026120,2006$.

[54] N. Przulj, "Biological network comparison using graphlet degree distribution," Bioinformatics, vol. 23, pp. 177-183, 2007.

[55] P. Zhao, J. Han, and Y. Sun, "Y.: P-rank: a comprehensive structural similarity measure over information networks,” in In: CIKM 2009: Proceeding of the 18th ACM conference on Information and knowledge management, 2009.

[56] L. Li, D. Alderson, J. C. Doyle, and W. Willinger, "Towards a theory of scale-free graphs: Definition, properties, and implications," Internet Mathematics, vol. 2, pp. 431-523, 2005.

[57] F. Mémoli and G. Sapiro, "A theoretical and computational framework for isometry invariant recognition of point cloud data," Found. Comput. Math., vol. 5, pp. 313-347, 2005.

[58] L. Hertz and H. Zielke, "Astrocytic control of glutamatergic activity: Astrocytes as stars of the show," Trends Neurosci., vol. 27, pp. 735-743, 2004.

[59] E. Kang, D. S. Lee, J. S. Lee, H. Kang, C. H. Hwang, S. H. Oh, C. S. Kim, J. K. Chung, M. C. Lee, M. J. Jang, Y. J. Lee, P. Morosan, and K. Zilles, "Developmental hemispheric asymmetry of interregional metabolic correlation of the auditory cortex in deaf subjects," NeuroImage, vol. 19, pp. 777-783, 2003.

[60] D. S. Lee, H. Kang, H. Kim, H. Park, J. S. Oh, J. S. Lee, and M. C. Lee, "Metabolic connectivity by interregional correlation analysis using statistical parametric mapping (SPM) and FDG brain PET; methodological development and patterns of metabolic connectivity in adults," Eur. J. Nucl. Med. Mol. Imaging, vol. 35, pp. 1681-1691, 2008.

[61] B. Vogt, L. Vogt, and S. Laureys, "Cytology and functionally correlated circuits of human posterior cingulate areas," NeuroImage, vol. 15, pp. 452-466, 2006.

[62] J. S. Lee, D. S. Lee, Y. K. Kim, J. S. Kim, J. M. Lee, B. B. Koo, J. J. Kim, J. S. Kwon, T. W. Yoo, K. H. Chang, S. I. Kim, H. Kang, E. Kang, J. K. Chung, and M. C. Lee, "Quantification of brain images using korean standard templates and structural and cytoarchitectonic probabilistic maps," Korean J. Nucl. Med., vol. 38, pp. 241-252, 2004.

[63] E. Courchesne, K. Pierce, C. M. Schumann, E. Redcay, J. A. Buckwalter, D. P. Kennedy, and J. Morgan, "Mapping early brain development in autism," Neuron, vol. 56, pp. 399-413, 2007.

[64] M. Dapretto, M. S. Davies, J. H. Pfeifer, A. A. Scott, M. Sigman, S. M. Bookheimer, and M. Iacoboni, "Understanding emotions in others: Mirror neuron dysfunction in children with autism spectrum disorders," Nat. Neurosci., vol. 9, pp. 28-30, 2005.

[65] C. J. Vaidya and M. Stollstorff, "Cognitive neuroscience of attention deficit hyperactivity disorder: current status and working hypotheses," Dev. Disabil. Res. Rev., vol. 14, pp. 261-267, 2008.

[66] J. P. Piek and M. J. Dyck, "Sensory-motor deficits in children with developmental coordination disorder, attention deficit hyperactivity disorder and autistic disorder," Hum. Movement Sci., vol. 23, pp. 475-488, 2004. 